Check for updates

Cite this: Chem. Commun., 2018, 54,3831

Received 6th March 2018,

Accepted 14th March 2018

DOI: $10.1039 / c 8 c c 01789 a$

rsc.li/chemcomm

\section{Black phosphorus with near-superhydrophobic properties and long-term stability in aqueous media†}

\author{
Peter D. Matthews, (D)*ab Wisit Hirunpinyopas, ${ }^{b c}$ Edward A. Lewis, ${ }^{d}$ Jack R. Brent, ${ }^{d}$ \\ Paul D. McNaughter, (DD ${ }^{b}$ Niting Zeng, ${ }^{d}$ Andrew G. Thomas, (D) d Paul O'Brien, (D) bd \\ Brian Derby, (D) Mark A. Bissett, (D) ${ }^{\text {cd }}$ Sarah J. Haigh, (D) ${ }^{\text {cd }}$ Robert A. W. Dryfe (D) ${ }^{b}$ \\ and David J. Lewis (D)*d
}

\begin{abstract}
Black phosphorus is a two-dimensional material that has potential applications in energy storage, high frequency electronics and sensing, yet it suffers from instability in oxygenated and/or aqueous systems. Here we present the use of a polymeric stabilizer which prevents the degradation of nearly $68 \%$ of the material in aqueous media over the course of ca. 1 month.
\end{abstract}

Black phosphorus (BP) has attracted attention as an elemental $2 \mathrm{D}$ material. ${ }^{1-5}$ The material is comprised of a plane of $\mathrm{sp}^{3}$ hybridised phosphorus atoms with tetrahedral bonding, which results in a puckered lattice containing 'armchair' and 'zig-zag' directions. This leads to in-plane anisotropy in the physical and electronic structure, which gives it intriguing photoelectronic and thermoelectric properties. ${ }^{6}$ Additionally, the band gap of BP can be tuned by simply varying the number of layers in each flake - giving control from $0.3 \mathrm{eV}$ (for bulk BP) to $1.5 \mathrm{eV}$ (for monolayer) with the additional benefit that the band gap transitions are all direct in nature and thus strongly interact with light. ${ }^{7,8}$ Additionally BP does not have to be monolayer to perform well in devices such as transistors - for example the carrier mobility of FETs as the active layer is in the range $10^{2}-10^{3} \mathrm{~cm}^{2} \mathrm{~V}^{-1} \mathrm{~s}^{-1}$ for the BP thickness range $10-50 \mathrm{~nm}$, and decreases exponentially below $10 \mathrm{~nm}$. Drain current in the same devices tends to peak around $10 \mathrm{~nm}$ BP thickness. ${ }^{3}$

This adjustability, coupled with the aforementioned anisotropy and high p-type carrier mobility make BP an exceptional candidate for sensing, energy storage, water splitting and high frequency electronics. ${ }^{9-15}$

\footnotetext{
${ }^{a}$ School of Chemical \& Physical Sciences, Keele University, Staffordshire, ST5 5BG, UK. E-mail: p.d.matthews@keele.ac.uk

${ }^{b}$ School of Chemistry, University of Manchester, Oxford Road, Manchester, M13 9PL, UK

${ }^{c}$ National Graphene Institute, University of Manchester, Oxford Road, M13 9PL, UK

${ }^{d}$ School of Materials, University of Manchester, Oxford Road, Manchester,

M13 9PL, UK. E-mail: david.lewis-4@manchester.ac.uk

$\dagger$ Electronic supplementary information (ESI) available: Includes detailed synthetic methodology and supplementary electron microscopy images. See DOI: $10.1039 / \mathrm{c} 8 \mathrm{cc} 01789 \mathrm{a}$
}

There are currently two major methods for the generation of nanomaterials: the so-called 'top-down' and 'bottom-up' approaches. For BP in particular, difficulties sourcing appropriate molecular precursors make 'bottom-up' methods such as chemical vapor deposition (CVD) refractory, ${ }^{16}$ and thus 'top-down' routes are, at this juncture, predominant. These processes generally involve the cleavage of crystalline bulk BP into individual layers, and the most successful subset of this methodology in terms of potential scalability for production of free standing sheets in solution for commercial exploitation is liquid phase exfoliation (LPE) ${ }^{17}$

Initial efforts to produce few-layer black phosphorus (FL-BP) by LPE made use of high boiling organic solvents, such as $N$-methyl-2-pyrrolidone (NMP), dimethylformamide (DMF), dimethyl sulfoxide (DMSO), and $N$-cyclohexyl-2-pyrrolidone (CHP), ${ }^{18-22}$ which were based on methods which had been generally successful for extant 2D materials such as graphene and h-BN. Indeed, the use of these solvents was shown by Hersam to impart greater stability in air compared with micromechanically (Scotch tape) exfoliated $\mathrm{BP}$, presumably due to surface adsorption of the residual solvent. ${ }^{20}$ However, the use of these high-boiling solvents is not particularly environmentally friendly, and are potentially hard to remove downstream in an industrial process, and also have drawbacks with use for biological applications due to toxicity. Therefore, attention moved to consider the use of aqueous media for processing BP by LPE.

However, BP is prone to hydrolysis to inorganic phosphate in aqueous media due to the energetic gain made by the formation of $\mathrm{P}=\mathrm{O}$ bonds by oxophilic phosphorus; Coleman et al. have demonstrated that black phosphorus nanosheets degrade in CHP doped with water. ${ }^{19}$ Hersam and co-workers reported that stable dispersions of BP may be obtained by LPE using sodium dodecyl sulfate (SDS) surfactant in aqueous media, and although no temporal stability data was presented, the material is stable within time-to-analysis. ${ }^{23}$ Zhang et al. have stabilized BP flakes on graphene oxide substrates ${ }^{24}$ or coating the surface of the BP with $\mathrm{Ag}^{+}$ions. ${ }^{25} \mathrm{We}$ have previously reported that exfoliation in aqueous $1 \%$ w/v Triton X-100 (TX-100, $\mathrm{C}_{14} \mathrm{H}_{22} \mathrm{O}\left(\mathrm{C}_{2} \mathrm{H}_{4} \mathrm{O}\right)_{n}$ where $\left.n=9-10\right)$ 
gives BP dispersions with an observed lifetime of $c a .500 \mathrm{~h}$ but unfortunately we also observed that the TX-100 stabilized BP sol in water experiences ca. $80 \%$ total degradation (i.e. only $20 \%$ remains as BP over time as-measured by a range of spectroscopic techniques such as ${ }^{31} \mathrm{P}$ NMR and inductively coupled plasma optical emission spectroscopy (ICP-OES), with the nanosheets degrading to phosphate species; we concluded that these materials have a 'shelf life' in terms of their potency. ${ }^{26}$ Therefore there is a need to produce stable dispersions of black phosphorus nanosheets in aqueous media that exhibit long term stability that can potentially be used for a myriad of new applications.

Recently we proposed, in a review article regarding solution processing and stability of black phosphorus, the use of extremely hydrophobic surfactants which may be able to protect BP in aqueous media. ${ }^{17}$ In this paper we now present a fluorinated surfactant, Zonyl ${ }^{\circledR} 7950$ (Z7950) which is used for exfoliation of $\mathrm{BP}$ in aqueous media, that leads to BP sols that are comparable in stability to BP dispersed in anhydrous organic solvents. We also immobilize this material on a PVDF membrane and show that the nanostructured composite exhibits near-superhydrophobic properties which are superior in hydrophobicity to that of Teflon.

We prepared suspensions of BP nanosheets by sonicating $100 \mathrm{mg}$ of bulk BP in $15 \mathrm{~mL}$ of a degassed aqueous solution containing $1 \% \mathrm{w} / \mathrm{v} \mathrm{Z7950}\left(\mathrm{MeC}\left(\mathrm{CH}_{2}\right) \mathrm{CO}_{2}\left(\mathrm{CH}_{2}\right)_{2}\left(\mathrm{CF}_{2}\right)_{n} \mathrm{CF}_{3}\right)$ for $36 \mathrm{~h}$ (ultrasonic bath, $820 \mathrm{~W}$ across four horns operating at $37 \mathrm{kHz}$ frequency and $30 \%$ power, cooled to $<30{ }^{\circ} \mathrm{C}$ throughout). This sample was then centrifuged at $1046 \mathrm{~g}$ (3000 rpm, rotor length $10.4 \mathrm{~cm}$ ) for $30 \mathrm{~min}$ to remove any unexfoliated material. Raman spectroscopy of the flakes show the characteristic optical modes of black phosphorus at 361, 438 and $465 \mathrm{~cm}^{-1}$ (Fig. 1a).

Atomic force microscopy (AFM) images show that the flakes are $17.1 \pm 2.1 \mathrm{~nm}(N=50)$ in height and $0.324 \pm 0.03 \mu \mathrm{m}(N=50)$
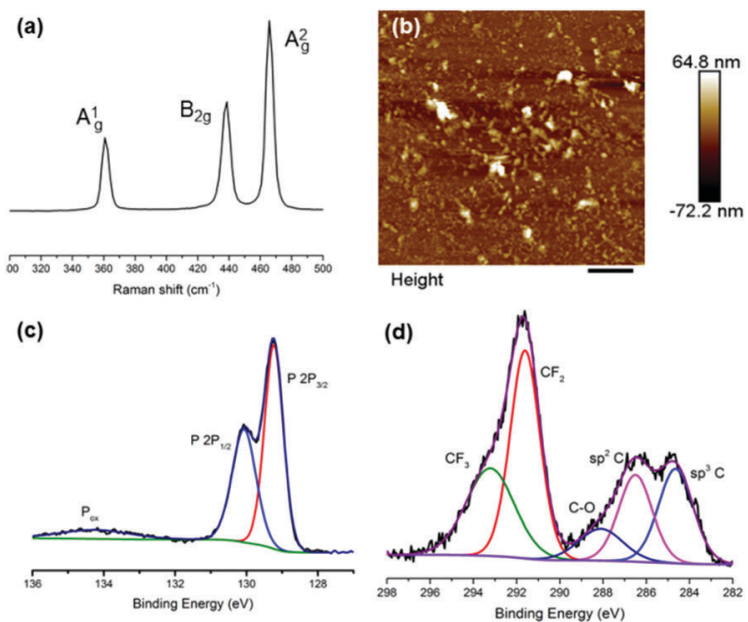

Fig. 1 Characterization of as-prepared BP nanosheets from liquid phase exfoliation of bulk BP in $1 \% \mathrm{w} / \mathrm{v}$ Zonyl 7950. (a) Raman spectrum of BP nanosheets showing the characteristic optical phonons at 361, 438 and $465 \mathrm{~cm}^{-1}$. (b) AFM of as-prepared BP nanosheets, with $z=17.1 \pm 2.1 \mathrm{~nm}$ $(N=50)$. (c) High resolution XP spectrum of the phosphorus region of BP nanosheets, showing minimal oxidized species (Pox). (d) High resolution $\mathrm{XP}$ spectrum of the carbon region of BP nanosheets, showing the presence of $\mathrm{CF}_{3}, \mathrm{CF}_{2}$ and $\mathrm{C}-\mathrm{O}$, all associated with the binding of $\mathrm{Z7950}$ to BP. in length (Fig. 1b and Fig. S3, S4, ESI $\dagger$ ). We note that the flakes in the images are not especially well defined, but this is to be expected owing to the presence of an organic surfactant on the surface of the flakes. We minimised the amount of surfactant residue by rinsing samples after deposition and included only those features which could unambiguously be considered nanoflakes, based on defined shapes and edges characteristic of inorganic particles rather than non-crystalline organics. This may have led to a slight overestimate of the average size as it is more difficult to identify extremely small particles.

X-ray photoelectron spectroscopy (XPS) characterization of the surface of the as-prepared flakes shows a number of interesting features in the $\mathrm{C}$ and $\mathrm{P}$ regions of the spectrum (Fig. 1c and $\mathrm{d}$ ). In the $\mathrm{P}$ region, there seems minimal oxidation of the BP material, bearing in mind the exfoliation is performed in water and we therefore suggest that hydrolysis of the BP has been minimised. In the $\mathrm{C}$ region, there is striking evidence of the fluorinated polymer bound to the surface of the BP nanosheets by the appearance of peaks corresponding to $\mathrm{CF}_{3}, \mathrm{CF}_{2}$ and $\mathrm{C}-\mathrm{O}$ groups of Zonyl 7950.

Electron microscopy shows that the obtained flakes are relatively large in size (Fig. 2c) and high resolution transmission electron microscope (HRTEM) images indicate that the flakes are high quality single crystals (Fig. 2a). The fast Fourier transforms (FFT) of atomic resolution images can be indexed to black phosphorus viewed along the [100] zone axis (Fig. 2b). Energy dispersive X-ray (EDX) spectrum imaging reveals that the fluorine signal is co-localized with the phosphorus signal, suggesting that the BP flakes are coated in Z7950, presumably by physisorption of the non-polar fluorine chains (Fig. $2 \mathrm{~d}$ and e).

We assessed the stability of the BP sheets over time by measuring the optical absorbance at $465 \mathrm{~nm}$ (Fig. 3). This established technique uses the light scattering of the BP colloid to assess the potency of the solution as phosphate degradation

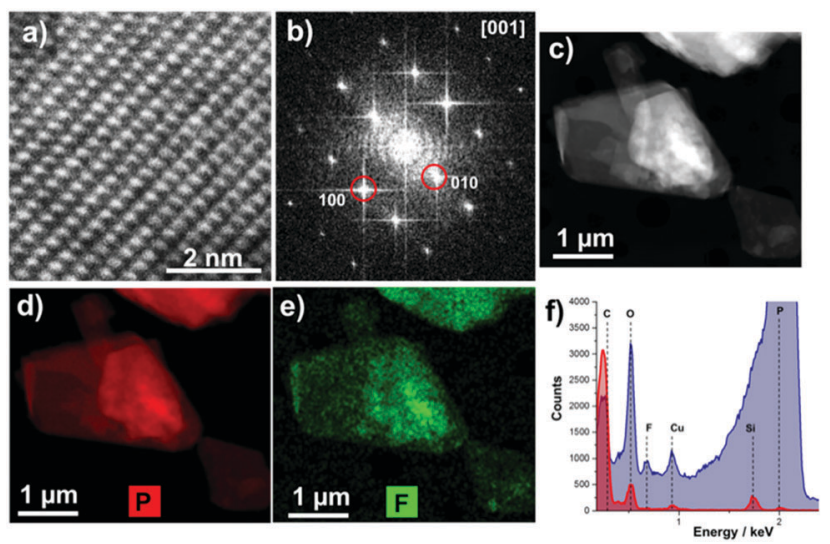

Fig. 2 (a) HRTEM image and (b) the associated fast Fourier transform (FFT). The FFT indexes to black phosphorus viewed down the [001] zone axis. (c) High angle annular dark field scanning transmission electron microscope (HAADF STEM) image of freshly prepared FL-BP. EDX spectrum imaging of the region shown in (c) was used to produce maps of $P(d)$ and $F(e)$ demonstrating colocalization of $P$ and $F$. (f) Comparison of spectra taken from the flake in (c) (shown in blue) and from the surrounding carbon support (shown in red), further demonstrate that $F$ is spatially co-localised with $P$. 


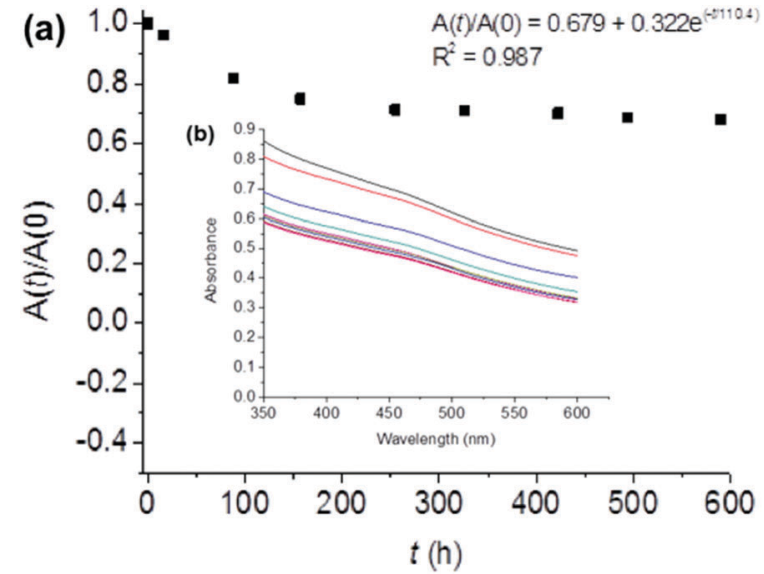

Fig. 3 Stability of BP flakes in solution. (a) The degradation of FL-BP flakes in aqueous $1 \% \mathrm{w} / \mathrm{v}$ Z7950 determined by the ratio of optical absorption at $465 \mathrm{~nm}$ at time $t$ and time zero. The observed lifetime from the fitted decay is around $110 \mathrm{~h}$, whilst $c a$. $68 \%$ of the flakes remains undegraded over a period of ca. 1 month in this media. Inset: Data fitting and $R^{2}$. (b) UV-Vis absorption data used to construct the plot in (a).

products do not absorb in this region of the electromagnetic spectrum. The degradation curve was fitted to the empirical first-order decay (eqn (1)) used previously by both Coleman et al. ${ }^{18}$ and Lewis and co-workers to assess solution phase BP degradation: ${ }^{23}$

$$
A_{t} / A_{0}=A_{\text {stable }}+A_{\text {unstable }} \mathrm{e}^{-\frac{t}{\tau}}
$$

where $A_{t}$ and $A_{0}$ are the absorbance at time $t$ and time zero respectively, $A_{\text {stable }}$ is the absorbance caused by flakes that do not degrade, $A_{\text {unstable }}$ is the absorbance caused by flakes that are degraded, $t$ is the time (in hours) and $\tau$ is therefore the observed lifetime of the nanosheets. It was found that this equation is a good fit to the absorbance data, corresponding to a lifetime of $110 \mathrm{~h}$, whilst $c a .68 \%$ of the flakes remain undegraded over the course of $650 \mathrm{~h}$ ( $c a .1$ month). This level of stability is unprecedented in an aqueous system, and is on par with that of anhydrous organic systems such as the BP-CHP system reported by Coleman and co-workers. ${ }^{19}$

Raman spectroscopy of the flakes after $650 \mathrm{~h}$ demonstrates the same shifts at 361,438 and $465 \mathrm{~cm}^{-1}$ as seen in the starting material (ESI, $\dagger$ Fig. S1). There was negligible decrease in the $A_{\mathrm{g}}^{1} / A_{\mathrm{g}}^{2}$ ratio observed ( $0.36 v s$. 0.34 ), and is well within the limits of this ratio for unoxidised material as-proposed by Favron et al. (0.2-0.6). ${ }^{27,28}$ High angle annular dark field scanning transmission electron microscope (HAADF STEM) images show that the flake height and width of the aged flakes is smaller than freshly exfoliated ones (ESI, $\dagger$ Fig. S1).

The processing route that we describe, with the characterization data alongside the stability data, strongly suggests that BP is stabilized in solution by adsorption of extremely hydrophobic Z7950 onto the phosphorus basal plane, which precludes the ingress of water and arrests degradation from hydrolytic pathways. Similarly, Beton and co-workers have recently shown that bulk BP is amenable to stabilization by the adsorption of polymeric networks upon its surface. ${ }^{29}$ Hirsch et al. have also

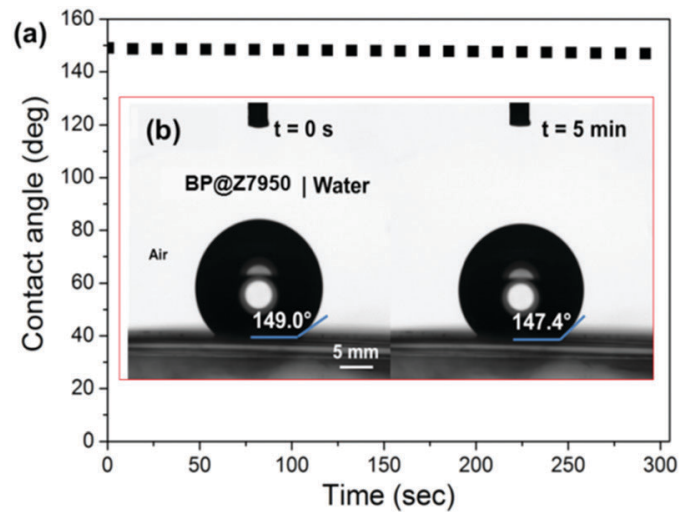

Fig. 4 Contact angle measurements on a PVDF membrane with BP immobilized at the surface. (a) Plot of contact angle $\left(^{\circ}\right)$ over time for a drop of deionized water on the BP supported by PVDF membrane. (b) Optical images (CCD camera) of the contact angle of a deionised water droplet on the BP-coated membrane at $t=0$ and $5 \mathrm{~min}$.

shown that adsorption of perylene diimide can stabilize BP by adsorption. ${ }^{30}$ We are currently investigating the mode of binding and how this relates to stabilization of BP.

We measured the water contact angle (WCA) of deionized water on the BP flakes to provide further evidence that the fluorinated surfactant forms a hydrophobic barrier on the flakes' surface. BP was immobilized and washed on a polyvinylidene fluoride (PVDF) membrane. The contact angle of deionized water on the BP-PVDF nanocomposite membrane was then measured (Fig. 4). The surface of the membrane initially displays a WCA in air of $c a .147 .9 \pm 0.5^{\circ}$ in three different areas, approaching super-hydrophobicity $\left(>150^{\circ}\right)$ as shown in Fig. 4a. The WCA on phosphorene was monitored as a function of time and decreased negligibly after 5 minutes as shown in Fig. $4 \mathrm{~b}$. This constant hydrophobicity, corresponds to the Cassie impregnation wetting model as a liquid is impregnated into a surface of the BP membrane. ${ }^{31,32}$ This indicates that the surface of the membrane has a nanoscale texture upon which the droplets sit, and explains why the phosphorene membrane has a WCA that is near-superhydrophobic and much greater than that of Teflon (polytetrafluoroethylene, WCA $\left.\sim 110^{\circ}\right)$. In longer tests $(t>10 \mathrm{~min})$ it was observed that the contact angle drops to $c a .100^{\circ}$ (ESI, $\dagger$ Fig. S2). We note that Ruoff and Sutter reported that the WCA of pristine BP is $99^{\circ}$, whilst oxidized material is hydrophilic $\left(\right.$ WCA $\left.\sim 4^{\circ}\right) .{ }^{33}$ We therefore tentatively suggest that this reduction in WCA over time corresponds to the Z7950 being washed off the flakes by the water droplet, revealing a close to pristine BP surface. Hence, not only does the surfactant stabilize the BP in solution thus allowing storage as a colloidal suspension, but the surfactant can be washed away to reveal pristine BP which is important for electronics applications as surfactants are usually insulating species that stymie device performance by isolation of the material from charge carriers and electrical contacts.

In conclusion, we have been able to stabilize few-layer black phosphorus in aqueous media through the addition of a fluorinated surfactant, Zonyl 7950. The obtained material has 
a decay constant of $110 \mathrm{~h}$, and after 4 weeks $c a$. $70 \%$ of the material remains stable in solution. We have shown through EDX and XPS spectroscopy and contact angle measurement that the flakes are coated in the surfactant. Immobilisation of this hydrophobic nanocomposite onto PVDF membranes leads to the formation of a near superhydrophobic membrane. We believe that the formation of this water stable solution of $\mathrm{BP}$ is a significant step to realizing some of the breathtaking potential applications of this exciting material.

The authors would like to acknowledge the EPSRC: Doctoral Prizes for P. D. M. and E. A. L., grant EP/M507969/1, award EP/R013446/1 to D. J. L., EP/K010298/1 to P. D. McN and P. O. B. Some of the equipment used in this study were provided by the EPSRC Core Capability in Chemistry grant (EP/K039547/1). P. D. M. would like to acknowledge the UK Government and European Union as contributors to the Smart Energy Network Demonstrator, ERDF project number 32R16P00706).

\section{Conflicts of interest}

There are no conflicts to declare.

\section{References}

1 R. A. Doganov, E. C. T. O'Farrell, S. P. Koenig, Y. Yeo, A. Ziletti, A. Carvalho, D. K. Campbell, D. F. Coker, K. Watanabe, T. Taniguchi, A. H. C. Neto and B. Özyilmaz, Nat. Commun., 2015, 6, 6647.

2 Z. Guo, H. Zhang, S. Lu, Z. Wang, S. Tang, J. Shao, Z. Sun, H. Xie, H. Wang, X. F. Yu and P. K. Chu, Adv. Funct. Mater., 2015, 25, 6996-7002.

3 L. Li, Y. Yu, G. J. Ye, Q. Ge, X. Ou, H. Wu, D. Feng, X. H. Chen and Y. Zhang, Nat. Nanotechnol., 2014, 9, 372-377.

4 X. Wang, A. M. Jones, K. L. Seyler, V. Tran, Y. Jia, H. Zhao, H. Wang, L. Yang, X. Xu and F. Xia, Nat. Nanotechnol., 2015, 10, 517-521.

5 F. Xia, H. Wang and Y. Jia, Nat. Commun., 2014, 5, 4458.

6 V. Tran, R. Soklaski, Y. Liang and L. Yang, Phys. Rev. B: Condens. Matter Mater. Phys., 2014, 89, 1-6.

7 H. Liu, Y. Du, Y. Deng and P. D. Ye, Chem. Soc. Rev., 2015, 44, 2732-2743.

8 H. Liu, A. T. Neal, Z. Zhu, Z. Luo, X. Xu, D. Tomanek and P. D. Ye, ACS Nano, 2014, 8, 4033-4041.

9 M. Buscema, D. J. Groenendijk, S. I. Blanter, G. A. Steele, H. S. van der Zant and A. Castellanos-Gomez, Nano Lett., 2014, 14, 3347-3352.

10 V. Kumar, J. R. Brent, M. Shorie, H. Kaur, G. Chadha, A. G. Thomas, E. A. Lewis, A. P. Rooney, L. Nguyen, X. L. Zhong, M. G. Burke, S. J. Haigh, A. Walton, P. D. McNaughter, A. A. Tedstone, N. Savjani, C. A. Muryn, P. O'Brien, A. K. Ganguli, D. J. Lewis and P. Sabherwal, ACS Appl. Mater. Interfaces, 2016, 8, 22860-22868.

11 W. Li, Y. Yang, G. Zhang and Y.-W. W. Zhang, Nano Lett., 2015, 15, 1691-1697.
12 M. Z. Rahman, C. W. Kwong, K. Davey and S. Z. Qiao, Energy Environ. Sci., 2016, 9, 709-728.

13 J. Sun, H.-W. Lee, M. Pasta, H. Yuan, G. Zheng, Y. Sun, Y. Li and Y. Cui, Nat. Nanotechnol., 2015, 10, 980-985.

14 Y. Zhang, H. Wang, Z. Luo, H. T. Tan, B. Li, S. Sun, Z. Li, Y. Zong, Z. J. Xu, Y. Yang, K. A. Khor and Q. Yan, Adv. Energy Mater., 2016, 6, 1600453.

15 X. Han, C. Liu, J. Sun, A. D. Sendek and W. Yang, RSC Adv., 2018, 8, 7196-7204.

16 X. Li, B. Deng, X. Wang, S. Chen, M. Vaisman, S. Karato, G. Pan, M. Larry Lee, J. Cha, H. Wang and F. Xia, 2D Mater., 2015, 2, 31002.

17 E. A. Lewis, J. R. Brent, B. Derby, S. J. Haigh and D. J. Lewis, Chem. Commun., 2017, 53, 1445-1458.

18 J. R. Brent, N. Savjani, E. A. Lewis, S. J. Haigh, D. J. Lewis and P. O'Brien, Chem. Commun., 2014, 50, 13338-13341.

19 D. Hanlon, C. Backes, E. Doherty, C. S. Cucinotta, N. C. Berner, C. Boland, K. Lee, A. Harvey, P. Lynch, Z. Gholamvand, S. Zhang, K. Wang, G. Moynihan, A. Pokle, Q. M. Ramasse, N. McEvoy, W. J. Blau, J. Wang, G. Abellan, F. Hauke, A. Hirsch, S. Sanvito, D. D. O'Regan, G. S. Duesberg, V. Nicolosi and J. N. Coleman, Nat. Commun., 2015, 6, 8563.

20 J. Kang, J. D. Wood, S. A. Wells, J. H. Lee, X. Liu, K. S. Chen and M. C. Hersam, ACS Nano, 2015, 9, 3596-3604.

21 A. H. Woomer, T. W. Farnsworth, J. Hu, R. A. Wells, C. L. Donley and S. C. Warren, ACS Nano, 2015, 9, 8869-8884.

22 P. Yasaei, B. Kumar, T. Foroozan, C. Wang, M. Asadi, D. Tuschel, J. E. Indacochea, R. F. Klie and A. Salehi-Khojin, Adv. Mater., 2015, 27, 1887-1892.

23 J. Kang, S. A. Wells, J. D. Wood, J.-H. Lee, X. Liu, C. R. Ryder, J. Zhu, J. R. Guest, C. A. Husko and M. C. Hersam, Proc. Natl. Acad. Sci. U. S. A., 2016, 113, 11688-11693.

24 C. Xing, G. Jing, X. Liang, M. Qiu, Z. Li, R. Cao, X. Li, D. Fan and H. Zhang, Nanoscale, 2017, 9, 8096-8101.

25 Z. Guo, S. Chen, Z. Wang, Z. Yang, F. Liu, Y. Xu, J. Wang, Y. Yi, H. Zhang, L. Liao, P. K. Chu and X. F. Yu, Adv. Mater., 2017, 29, 1703811.

26 J. R. Brent, A. K. Ganguli, V. Kumar, D. J. Lewis, P. D. Mcnaughter and P. O. Brien, RSC Adv., 2016, 6, 86955-86958.

27 G. Abellán, S. Wild, V. Lloret, N. Scheuschner, R. Gillen, U. Mundloch, J. Maultzsch, M. Varela, F. Hauke and A. Hirsch, J. Am. Chem. Soc., 2017, 139, 10432-10440.

28 A. Favron, E. Gaufrès, F. Fossard, A.-L. Phaneuf-L'Heureux, N. Y.-W. Tang, P. L. Lévesque, A. Loiseau, R. Leonelli, S. Francoeur and R. Martel, Nat. Mater., 2015, 14, 826-832.

29 V. V. Korolkov, I. G. Timokhin, R. Haubrichs, E. F. Smith, L. Yang, S. Yang, N. R. Champness, M. Schröder and P. H. Beton, Nat. Commun., 2017, 8, 1385.

30 G. Abellán, V. Lloret, U. Mundloch, M. Marcia, C. Neiss, A. Görling, M. Varela, F. Hauke and A. Hirsch, Angew. Chem., Int. Ed., 2016, 55, 14557-14562.

31 E. Bormashenko, T. Stein, R. Pogreb and D. Aurbach, J. Phys. Chem. C, 2009, 113, 5568-5572.

32 L. Feng, Y. Zhang, J. Xi, Y. Zhu, N. Wang, F. Xia and L. Jiang, Langmuir, 2008, 24, 4114-4119.

33 Y. Huang, J. Qiao, K. He, S. Bliznakov, E. Sutter, X. Chen, D. Luo, F. Meng, D. Su, J. Decker, W. Ji, R. S. Ruoff and P. Sutter, Chem. Mater., 2016, 28, 8330-8339. 